\title{
Disease Response or Clinical Classification Previous Occurrence
}

National Cancer Institute

\section{Source}

National Cancer Institute. Disease Response or Clinical Classification Previous

Occurrence. NCI Thesaurus. Code C162321.

An indication or description that a particular disease response or clinical classification was obtained in the past. 